\title{
ANALYSES OF LANGUAGES ACCEPTED BY VARIETOR MACHINES
} IN CATEGORY

V. TRNKOVÁ

Faculty of Mathematics and Physics, Charles University, Prague, Czechoslovalia

$$
\text { J. AD } A ́ M E K
$$

Faculty of Electrical Engineering, Technical University, Prague, Czechoslovakia

\section{Introduction}

Languages accepted by finite sequential machines are characterized by the well-known Kleene theorem: they are precisely rational languages, i.e. those obtained from finite languages by a finite succession of operations $\cup$ (union), · (concatenation) and * (iteration). How far can this beautiful result be extended? Thatcher and Wright [7] present a generalization for tree machines: here not one concatenation and one iteration are sufficient, but rather a system of concatenations and corresponding iterations is considered. The internal reason for this is that in the case of tree machines it is not sufficient (as for sequential ones) to study machines with one initial state, but more initial states must be taken into account, and each initial state plays an individual role: it is important which initial states were used for a given tree to be accepted.

In the present paper we consider a very general situation: machines in a category in the sense of Arbib and Manes [4]. We define acceptors and finite acceptors on the one hand, and rational languages on the other hand. The main result is that every language accepted by a finite acceptor is rational. There is no hope for the converse (rational $\Rightarrow$ acceptable) to hold. In fact, even in the variety $\mathscr{V}$ of groupoids, defined by the equation

$$
x \cdot x=y \cdot y,
$$

the Kleene theorem fails to hold for $\mathscr{V}$-machines (i.e., binary tree machines in $\mathscr{V}$ ), see [8]. It remains open even for varieties of tree machines under which circumstances the Kleene theorem holds. 
The definition of languages and their concatenation which we present might seem technical at first, but it is justified by the motivating example of sequential machines with several initial states. This motivation is exhibited in [2], where the present result was announced; therefore we skip it here and start with the definitions needed. Let us remark that, at least, it suffices to consider one concatenation and one iteration here, not as in the case of tree machines (where, on the other hand, the definition of these operations is very natural and not technical at all).

The present paper is a part of a broad program of investigating the languages of (deterministic and non-deterministic) varietor machines, see [2], [3], [8], [9], [10].

\section{Accepted and rational languages}

1.1. Throughout the paper we assume that a category $\mathscr{K}$ is given together with its factorization system $(\mathscr{E}, \mathscr{M})$ and a functor $F: \mathscr{K} \rightarrow \mathscr{K}$ subject to conditions $\mathrm{O1-C4}$ below. Let us recall (e.g. from [4]) that an algebra of type $F$, shortly an $F$-algebra, is an arrow $F Q \stackrel{\delta}{\rightarrow} Q$ and a homomorphism from $F Q \stackrel{\delta}{\rightarrow} Q$ into $F Q^{\prime} \stackrel{\stackrel{\prime}{\prime}^{\prime}}{\rightarrow} Q^{\prime}$ is a morphism $f: Q \rightarrow Q^{\prime}$ in $\mathscr{K}$ for which the following diagram commutes:

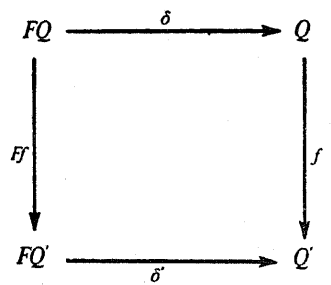

A free $F$-algebra, generated by an object $X$, is an $F$-algebra $F X^{\#} \stackrel{\oplus}{\rightarrow} X$ together with an arrow $X \stackrel{\eta}{\rightarrow} X^{\#}$, universal in the usual sense: for each algebra $F Q \stackrel{\delta}{\rightarrow} Q$ and each morphism $k: X \rightarrow Q$ there exists a unique "free extension" homomorphism $k^{*}:\left(X^{\#}, \varphi\right) \rightarrow(Q, \delta)$ with $k_{i}=\eta \cdot k^{*}$.

The conditions we require throughout are as follows:

C1. $F$ is a varietor, i.e., every object generates a free algebra.

C2. $F$ preserves $\mathscr{E}$, i.e. $e \in \mathscr{E}$ implies $F e \in \mathscr{E}$.

C3. $\mathscr{K}$ has finite limits and countable colimits.

C4. Pullback condition: opposite an $\mathscr{E}$-epi in any pullback there is an $\mathscr{E}$-epi.
1.2. Modifying the definition of machines of type $F$, due to Arbib and Manes, we define an acceptor of type $F$. This is an $F$-algebra with two subobjects (of initial and terminal states, respectively). Thus, an acceptor is a. 6 -tuple

$$
A=(Q, \delta, I, i, T, t),
$$

where $Q$ is an object (of states) in $\mathscr{K}, \delta: F Q \rightarrow Q$ is a morphism ("nextstate map"), and $i: I \rightarrow Q, t: T \rightarrow Q$ are morphisms in $\mathscr{M}$. We can extend $i$ freely to a homomorphism $i^{*}:\left(I^{\#}, \varphi\right) \rightarrow(Q, \delta)$, called the run map of $A$ :

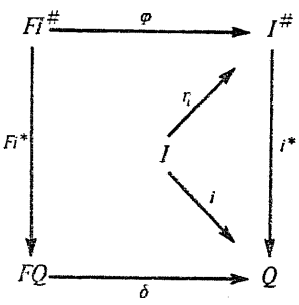

The language accepted by $A$ is defined as the preimage of the terminalstate subobject under the run map, i.e., the subobject $\lambda: L \rightarrow I^{\#}$ in the following pullback:

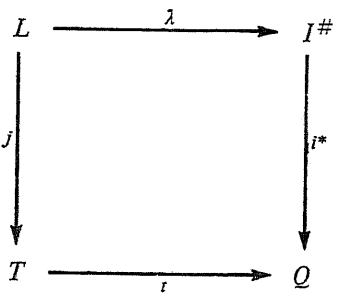

(since $t \in \mathscr{M}$ implies $\lambda \in \mathscr{M}$, this is indeed a subobject of $I^{\#}$ ).

1.3. In the present paper we shall make an extensive use of the calculus of relations in a category, see [5]. A relation from $A$ to $B$ ( $A, B$ objects of $\mathscr{K}$ ) is any $\mathscr{M}$-subobject of $A \times B$. A relation is determined by a pair of arrows $T \stackrel{f}{\rightarrow} A, T \stackrel{g}{\rightarrow} B$ if the image of the induced arrow $T \rightarrow A \times B$ is this relation. We then write $[f, g]: A \rightarrow B$. Every relation has a number of determining pairs. For example, given $e: T_{1} \rightarrow T$ in $\mathscr{E}$, we have

$$
[f, g]=[e \cdot f, e \cdot g]: A \rightarrow B .
$$


The composition of relations $[f, g]: A \rightarrow B$ and $[h, k]: B \rightarrow C$ is defined via the pullback of $g$ and $h$,

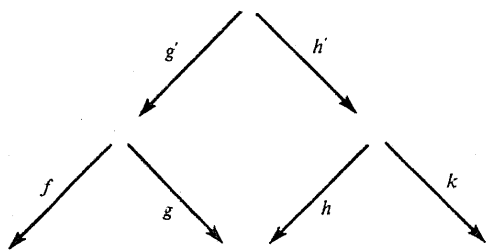

as follows:

$$
[f, g] \circ[h, k]=\left[g^{\prime} \cdot f, h^{\prime} \cdot k\right]: A \rightarrow C .
$$

The pullback condition (C4 in 1.1) guarantees that this definition is correct, i.e. independent of the choice of the representatives $f, g$ and $h, k$, see [5]. Evidently, it is associative.

1.4. Given two objects $I, J$ in $\mathscr{K}$, an extended language (or just language) from $I$ to $J$ is a relation $[f, g]: I^{\#} \rightarrow J$. We denote it by

$$
[f, g]: I \mapsto J .
$$

1.5. Let $A=(Q, \delta, I, i, T, t)$ and $\lambda, j$ be as in 1.2 .

Defintion. The extended language of an acceptor $A$ is

$$
L(A)=[\lambda, j]: I \mapsto T .
$$

1.6. For each morphism $f: X \rightarrow Y$ in $\mathscr{K}$ there exists a free extension of $f \cdot \eta_{Y}: X \rightarrow Y^{\#}$ to a homomorphism, denoted by

$$
f^{\#}:\left(X^{\#}, \varphi_{X}\right) \rightarrow\left(Y^{\#}, \varphi_{Y}\right) \text {. }
$$

Thus, $f^{\#}=\left(f \cdot \eta_{\bar{Y}}\right)^{*}$. Given an extended language $[f, g]: I \mapsto J$, we define an extended language

$$
[f, g]^{\#}=\left[f^{*}, g^{\#}\right]: I \mapsto J^{\#} .
$$

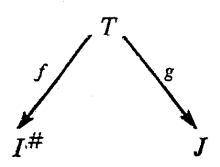

$L$

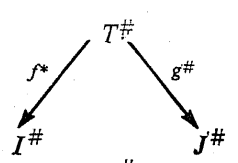

$L^{\#}$

\subsection{Rational operations on extended languages}

A) Union $\left[\alpha_{1}, \beta_{1}\right] \cup\left[\alpha_{2}, \beta_{2}\right]$ is defined whenever these languages have a joint domain $I$ and range $J$; this is the union of $\mathscr{M}$-subobjects of $I^{\#} \times J$.

Since $\mathscr{K}$ has finite sums, it has finite unions of $\mathscr{M}$-subobjects; the union of subobjects, represented by

$$
m_{1}: U_{1} \rightarrow V \text { and } m_{2}: U_{2} \rightarrow V \text { in } \mathscr{M},
$$

is the image of the induced morphism $\sigma_{1}+U_{2} \rightarrow V$.

B) Concatenation $\left[\alpha_{1}, \beta_{1}\right] \odot\left[\alpha_{2}, \beta_{2}\right]$ is defined whenever the range of $\left[a_{1}, \beta_{1}\right]$ equals the domain of $\left[a_{2}, \beta_{2}\right]$. Thus, let

$$
\left[\alpha_{1}, \beta_{1}\right]: I \mapsto J \quad \text { and } \quad\left[\alpha_{2}, \beta_{2}\right]: J \mapsto K
$$

be languages; we then define

$$
\begin{gathered}
{\left[\alpha_{1}, \beta_{1}\right] \odot\left[\alpha_{2}, \beta_{2}\right]=\left[\alpha_{1}, \beta_{1}\right]^{\#} \circ\left[\alpha_{2}, \beta_{2}\right]: I_{1} \mapsto K,} \\
{\left[\alpha_{1}, \beta_{1}\right]^{\#} \circ\left[\alpha_{2}, \beta_{2}\right]=\left[\tilde{\alpha}_{2} \cdot \alpha_{1}^{*}, \tilde{\beta}_{1} \cdot \beta_{2}\right] .}
\end{gathered}
$$

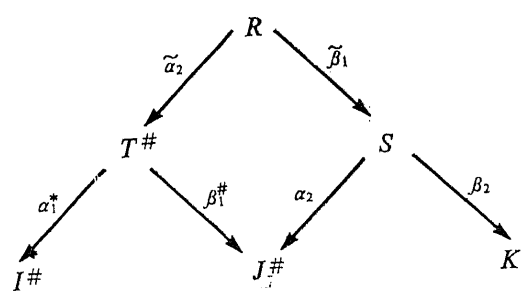

C) Iteration $[\alpha, \beta]^{*}$ is defined whenever the domain and range of $[\alpha, \beta]$ are equal. Given $[\alpha, \beta]: I \mapsto I$, define $[\alpha, \beta]^{*}: I \mapsto I$ by

$$
[\alpha, \beta]^{*}=\Lambda \cup[\alpha, \beta] \cup([\alpha, \beta] \odot[\alpha, \beta]) \cup(([\alpha, \beta] \odot[\alpha, \beta]) \odot[\alpha, \beta]) \ldots
$$

where $\Lambda=\left[\eta_{I}, 1_{I}\right]: I \mapsto I$.

Again, since $\mathscr{K}$ has countable colimits, $[\alpha, \beta]^{*}$ is well defined.

1.8. Let $\mathscr{F}$ bo a fixed class of objects of $\mathscr{K}$; we call its elements finite objects. In the present context no hypothesis on $\mathscr{F}$ is needed.

An acceptor $A=(Q, \delta, I, i, T, t)$ is called finite if its state object $Q$ as well as objects $I$ and $T$ are finite. A language $I \mapsto J$ is finite if it is determined by a pair

$$
f: M \rightarrow I^{\#}, \quad g: M \rightarrow J
$$

with a finite $M$. 
1.9. Definimion. An extended language is said to be rational if it can be expressed by rational operations $U, \odot, *$ used finitely many times on finite extended languages.

\section{The main theorem}

2.1. Algorithmic varietors. In a number of situations, free $F$-algebras $\left(X^{\#}, \varphi\right)$ can be obtained by a natural construction, called the free-algebra algorithm in [1]. First, define a sequence of objects $W_{n}$ and morphisms $k_{n}: W_{n} \rightarrow W_{n+1}$ by induction

(a) $W_{0}=X, W_{1}=X+F X$ and $k_{0}: X \rightarrow X+F X$ is canonical;

(b) $W_{n+1}=X+F W_{n}$ and $k_{n+1}=1_{X}+F k_{n}$

$$
\begin{aligned}
& X \stackrel{k_{0}}{\longrightarrow} X+F X \stackrel{{ }^{1} X^{+} F k_{0}}{\longrightarrow} X+F(X+F X) \stackrel{{ }^{1} X+F\left(1_{X}+F k_{0}\right)}{\longrightarrow} \ldots \rightarrow X^{\#}
\end{aligned}
$$

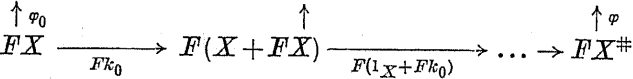

Denote by $X^{\#}$ and $w_{n}: W_{n} \rightarrow X^{\#}(n=0,1,2, \ldots)$ the colimit of this sequence. Since we have a canonical injection $F W_{n} \rightarrow W_{n+1}=X+F W_{n}$, we obtain a natural morphism $\varphi: F X^{\#} \rightarrow X^{\#}$ provided that also $F X^{\#}$ and $F w_{n}: F W_{n} \rightarrow F X^{\#}$ is a colimit of the sequence $\left\{F k_{n}\right\}_{n=0}^{\infty}$. Put $\eta=w_{0}$ : $X \rightarrow X^{\#}$; then $\left(X^{\#}, \varphi\right)$ is a free $F$-algebra, see [1].

DEFINITION. A functor $F$ is called an algorithmic varietor if it preserves the colimit of the above sequence $\left\{k_{n}\right\}_{n=0}^{\infty}$ for each object $X$.

Examples. Given a type $\Omega=\left\{\omega_{i}\right\}_{i \in I}$ of algebras, i.e. a collection of cardinals, denote by $F_{\Omega}$ the functor with $F_{\Omega} X=\prod_{i \in I} X^{\omega_{i}}$ (where $X^{\omega_{i}}$ denotes the product of $\omega_{i}$ copies of $X$ ), defined naturally on morphisms, $F_{\Omega} f=\coprod_{i \in I} f^{\omega_{i}}$. In sets, $F_{\Omega}$-algebras are precisely universal algebras of type $\Omega$, and homomorphisms also agree. Then $F_{\Omega}$ is an algorithmic varietor iff $\Omega$ is a finitary type.

In contrast, (a) every endofunctor of the category of countable sets and mappings is an algorithmic varietor [2]; (b) every functor $F_{0}$ on the category of $\omega$-complete posets and $\omega$-continuous maps is an algorithmic varietor [6].

2.2. Convention. Given morphisms $f: A \rightarrow C$ and $g: B \rightarrow C$, we denote by $f+g: A+B \rightarrow C$ the obvious induced morphism.

Let $F$ be an algorithmic varietor. Given an $F$-algebra $F Q \stackrel{\circ}{\rightarrow} Q$ and a morphism $f: X \rightarrow Q$, its free extension to a homomorphism $f^{*}:\left(X^{\#}, \varphi\right)$ $\rightarrow(Q, \delta)$ can be described as follows. Put

$$
f_{0}=f: W_{0} \rightarrow Q
$$

given $f_{n}: W_{n} \rightarrow Q$, define

$$
f_{n+1}=f \dot{+} F f_{n} \cdot \delta\left(X+F W_{n}=W_{n+1} \rightarrow Q\right) .
$$

Then the (unique) morphism $f^{*}: X^{\#} \rightarrow Q$ with

$$
w_{n} \cdot f^{*}=f_{n} \quad(n=0,1,2, \ldots)
$$

is a homomorphism and, of course, $\eta \cdot f^{*}\left(=w_{0} \cdot f^{*}\right)=f$. See [1].

2.3. To formulate the main result of our paper, we shall introduce the following languages for any acceptor $A=(Q, \delta, I, i, T, t)$

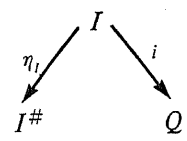

$D_{A}$

$$
\begin{aligned}
D_{A} & =\left[\eta_{I}, i\right]: I \mapsto Q, \\
M_{A} & =\left[F \eta_{Q} \cdot \varphi, \delta\right]: Q \mapsto Q, \\
R_{A} & =\left[t \cdot \eta_{Q}, 1_{T}\right]: Q \mapsto T .
\end{aligned}
$$
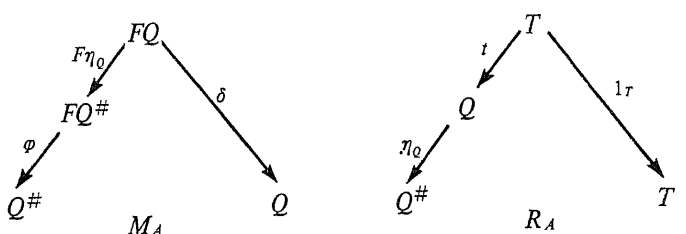

Let us mention that if $A$ is a finite acceptor, then $D_{A}, R_{A}$ are finite languages and $M_{A}$ is finite whenever $F Q$ is finite, which is so e.g. whenever $F$ preserves finite objects (i.e. $Q \in \mathscr{F}$ implies $F Q \in \mathscr{F}$ ).

2.4. Theorem on analyses. Let $F$ be an algorithmic varietor. For any acceptor $A=(Q, \delta, I, i, T, t)$, the language $L(A)$ accepted by $A$ fulfils

$$
L(A)=D_{A} \odot\left(\left(\Lambda \cup M_{A}\right)^{*} \odot R_{A}\right) .
$$

2.5. COROLLARY. If $F$ is an algorithmic varietor preserving finite objects, then every language accepted by a finite acceptor is rational.

The rest of our paper is devoted to the proof of 2.4 .

\section{Auxiliary propositions}

3.1. Analyses of iteration of any language $[\alpha, \beta]: I \mapsto I$ containing $\Lambda=\left[\eta_{I}, \mathbf{1}_{I}\right]$.

If $\alpha: P_{1} \rightarrow I^{\#}$ and $\beta: P_{1} \rightarrow I$ are the representing morphisms, we define morphisms

$$
\alpha_{n}: P_{n} \rightarrow P_{n-1}^{\#} \quad \text { and } \quad \beta_{n}: P_{n} \rightarrow P_{n-1}
$$


using pullbacks:

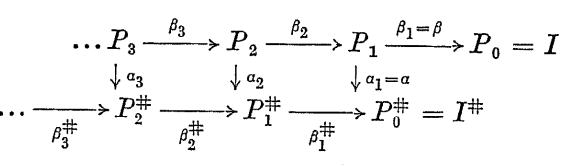

First, put

$$
\alpha_{1}=\alpha \text { and } \beta_{1}=\beta ;
$$

then let $\alpha_{2}, \beta_{2}$ be the pullback of $\beta_{1}^{\#}$ and $\alpha_{1}$; let $\alpha_{3}, \beta_{3}$ be the pullback of $\beta_{2}^{\#}$ and $\alpha_{2}$, etc. Put also $\beta_{0}=1_{I} ; \alpha_{0}=\eta_{I}$.

Further, define a sequence $e_{n}: P_{n} \rightarrow P_{n+1}$ with $e_{0}=1_{I}$ and

$$
e_{n} \cdot a_{n}=\eta_{P_{n-1}} \quad \text { and } \quad e_{n} \cdot \beta_{n}=\beta_{n-1} \cdot e_{n-1} \quad(n=1,2,3, \ldots)
$$

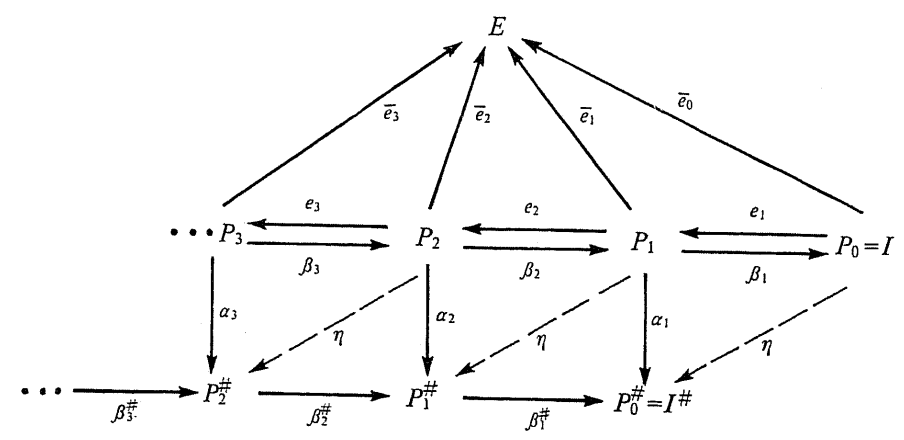

First, since $[\alpha, \beta]$ contajns $A=\left[\eta_{I}, 1_{I}\right]$, there exists an $e_{1}: I \rightarrow P$ with

$$
e_{1} \cdot \alpha_{1}=\eta_{I} \quad \text { and } \quad e_{1} \cdot \beta_{1}=1_{I}=\beta_{0} \cdot e_{0} .
$$

Now, $\eta_{P_{1}} \cdot \beta_{1}^{\#}=\beta_{1} \cdot \eta_{I}$ (by the definition of \#, see 1.6 ); therefore $\eta_{P_{1}} \cdot \beta_{1}^{\#}$ $=\left(\beta_{1} \cdot e_{1}\right) \alpha_{1}$ and, since $\beta_{1}^{\#}$ and $\alpha_{1}$ have pullback $\alpha_{2}, \beta_{2}$, there exists a unique $e_{2}: P_{1} \rightarrow P_{2}$ with

$$
e_{2} \cdot \alpha_{2}=\eta_{P_{1}} \quad \text { and } \quad e_{2} \cdot \beta_{2}=\beta_{1} \cdot e_{1} .
$$

Analogously we obtain $e_{3}, e_{4}, \ldots$

Denote by $E$, and $\bar{e}_{n}: P_{n} \rightarrow E$, the colimit of this sequence $\left\{e_{n}\right\}_{n=1}^{\infty}$.

3.2. Proposition. For any language $[\alpha, \beta]: I_{1} \mapsto$ containing $\Lambda$, we have

$$
[a, \beta]^{*}=[\bar{a}, \bar{\beta}],
$$

where $\bar{a}: E \rightarrow I^{*}$ is defined by $\bar{a} \cdot \bar{e}_{n}=a_{n} \cdot a_{n-1}^{*} \cdot a_{n-2}^{*} \cdot \ldots \cdot a_{1}^{*}$ and $\beta: E$ $\rightarrow I$ is defined by $\bar{\beta} \cdot \bar{e}_{n}=\beta_{n} \cdot \ldots \cdot \beta_{1}$.
Proof. (a) Morphisms $\alpha_{n} \cdot a_{n-1}^{*} \cdot \ldots \cdot a_{1}^{*}: P_{n} \rightarrow I^{\#}$ form a compatible family for $\left\{e_{n}\right\}$, because $e_{n} \cdot a_{n}=\eta$ implies

$$
e \cdot\left(a_{n} \cdot a_{n-1}^{*} \cdot \ldots \cdot a_{1}^{*}\right)=\eta \cdot a_{n-1}^{*} \cdot a_{n-2}^{*} \cdot \ldots \cdot a_{1}^{*}=a_{n-1} \cdot a_{n-2}^{*} \cdot \ldots \cdot a_{1}^{*} .
$$

Hence, $\bar{a}$ is correctly defined.

(b) Morphisms $\left(\beta_{n} \cdot \beta_{n-1} \cdot \ldots \cdot \beta_{1}\right): P_{n} \rightarrow I^{\text {\# }}$ form a compatible family for $\left\{e_{n}\right\}$ because $e_{k} \cdot \beta_{k}=\beta_{k-1} \cdot e_{k-1}$ implies

$$
\begin{aligned}
e_{n} \cdot\left(\beta_{n} \cdot \beta_{n-1} \cdot \ldots \cdot \beta_{1}\right)= & \beta_{n-1} \cdot e_{n-1} \cdot \beta_{n-1} \cdot \ldots \cdot \beta_{1} \\
= & \beta_{n-1} \cdot \beta_{n-2} \cdot e_{n-2} \cdot \beta_{n-2} \cdot \ldots \cdot \beta_{1} \\
& \cdots \cdots \cdots \cdots \cdots \\
= & \beta_{n-1} \cdot \beta_{n-2} \cdot \ldots \cdot \beta_{1} \cdot e_{0} \\
= & \beta_{n-1} \cdot \beta_{n-2} \cdot \ldots \cdot \beta_{1} .
\end{aligned}
$$

Hence, $\bar{\beta}$ is correctly defined.

(c) $[\alpha, \beta]^{*}=[\bar{\alpha}, \bar{\beta}]$. Indeed, we have

$$
[\alpha, \beta]^{*}=[\alpha, \beta] \cup([\alpha, \beta] \odot[\alpha, \beta]) \cup(([\alpha, \beta] \odot[\alpha, \beta]) \odot[\alpha, \beta]) \ldots
$$

and

1. $[\alpha, \beta]=\left[\alpha_{1}, \beta_{1}\right]$,

2. $[\alpha, \beta] \odot[\alpha, \beta]=\left[\alpha_{2} \cdot a_{1}^{*}, \beta_{2} \cdot \beta_{1}\right]$

as seen from $[\alpha, \beta] \odot[\alpha, \beta]=\left[\alpha_{1}^{*}, \beta_{1}^{\#}\right] \circ\left[\alpha_{1}, \beta_{1}\right]$ and:

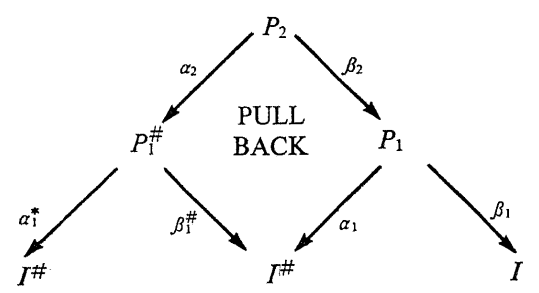

3. $([\alpha, \beta] \odot[\alpha, \beta]) \odot[\alpha, \beta]=\left[\alpha_{3} \cdot \alpha_{2}^{*} \cdot \alpha_{1}^{*}, \beta_{3} \cdot \beta_{2} \cdot \beta_{1}\right]$, which is equaly easy to see. Etc.

Thus, we see that

$$
[\alpha, \beta]^{*}=\bigcup_{n=1}^{\infty}\left[\bar{\alpha} \cdot \bar{e}_{n}, \bar{\beta} \cdot \bar{e}_{n}\right]=[\bar{\alpha}, \bar{\beta}] .
$$

3.3. For an arbitrary $F$-algebra $(Q, \delta)$ there exists a unique homomorphism $r:\left(Q^{\#}, \varphi\right) \rightarrow(Q, \delta)$ with $\eta \cdot r=1_{Q}$ (viz. $\left.r=\left(1_{Q}\right)^{*}\right)$. This gives 
rise to a language

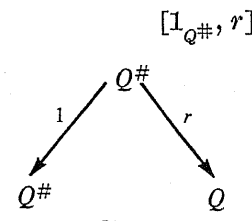

$[1, r]$

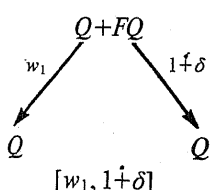

$\left[w_{1}, 1+\delta\right]$
The aim of the rest of this section is to show that this language is a result of iteration of the language

More precisely:

$$
\left[w_{1}, \mathbf{1}_{Q}+\delta\right]: Q \mapsto Q .
$$

3.4. Proposition. If $F$ is an algorithmic varietor, then for any $F$ algebra $F Q \stackrel{\circ}{\rightarrow} Q$ we have

$$
\left[w_{1}, 1_{Q}+\delta\right]^{*}=\left[1_{Q \#}, r\right]: Q \mapsto Q .
$$

Proof. Apply 3.1 to $[\alpha, \beta]=\left[w_{1}, 1_{Q}+\delta\right]$. First, this language contains $A$ because for $e_{1}=k_{0}: Q \rightarrow Q+F Q$ we have $k_{0} \cdot w_{1}=w=\eta_{Q}$ and $k_{0} \cdot\left(1_{Q}+\delta\right)=1_{Q}$. Thus, by 3.2,

$$
\left[w_{1}, 1_{Q}+\delta\right]^{*}=[\bar{\alpha}, \bar{\beta}] \text {. }
$$

We shall prove that (a) $\bar{a} \cdot r=\bar{\beta}$ and (b) $\bar{a} \in \mathscr{E}$. The latter implies $\left[1_{Q} \neq\right.$ 护,$\left.r\right]$ $=\left[\bar{a} \cdot 1_{Q \#}, \bar{\alpha} \cdot r\right] ;$ therefore $\left[1_{Q \#}, r\right]=[\bar{\alpha}, \bar{\beta}]$.

(a) We shall verify that

$$
\text { (*) } \quad\left(a_{n}^{*} \cdot \ldots \cdot \alpha_{1}^{*}\right) \cdot r=\left(\beta_{n} \cdot \ldots \cdot \beta_{1}\right)^{\#} \cdot r \quad \text { for } \quad n=1,2,3, \ldots \text {; }
$$

then for each $n$

$$
\begin{aligned}
\bar{e}_{n} \cdot(\bar{\alpha} \cdot r) & =a_{n} \cdot a_{n-1}^{*} \cdot \ldots \cdot a_{1}^{*} \cdot r & & (\text { definition of } \bar{\alpha}) \\
& =\eta \cdot a_{1}^{*} \cdot a_{n-1}^{*} \cdot \ldots \cdot a_{1}^{*} \cdot r & & \left(a_{n}=\eta \cdot a_{n}^{*}\right) \\
& =\eta \cdot\left(\beta_{n} \cdot \ldots \cdot \beta_{1}\right)^{\#} \cdot r & & (\text { by }(*)) \\
& =\left(\beta_{n} \cdot \ldots \cdot \beta_{1}\right) \cdot \eta \cdot r & & \left(\eta \cdot(-)^{\#}=(-) \cdot \eta\right) \\
& =\beta_{n} \cdot \ldots \cdot \beta_{1}=\bar{e}_{n} \cdot \bar{\beta} & & (\eta \cdot r=1 ; \text { definition of } \bar{\beta}) .
\end{aligned}
$$

There follows $\bar{a} \cdot r=\bar{\beta}$.

(A) $n=1$. We have $a_{1}=w_{1}=w_{0}+F w_{0} \cdot \varphi$ and, since $r$ is a homomorphism extending $1_{Q}$, also $\eta_{Q} \cdot r=1_{Q}$ and $\varphi \cdot r=F r \cdot \delta$. Thus

$$
\alpha_{1} \cdot r=\left(w_{0} \cdot r\right)+\left(F w_{0} \cdot \varphi \cdot r\right)=1_{Q}+\left(F\left(w_{0} \cdot r\right) \cdot \delta\right)=1_{Q}+\delta=\beta_{1} .
$$

Hence, $a_{1}^{*} \cdot r$ and $\beta_{1}^{\# \cdot r}$ are homomorphisms from $\left((Q+F Q)^{\#}, \varphi\right)$ which agree on $Q+F Q$ (for, $\eta_{Q+F Q} \cdot \alpha_{1}^{*} \cdot r=\alpha_{1} \cdot r$ while $\eta_{Q+F Q} \cdot \beta_{1}^{\#} \cdot r=\beta_{1} \cdot \eta_{Q} \cdot r=\beta_{1}$ ). Hence $\alpha_{1}^{*} \cdot r=\beta_{1}^{\#} \cdot r$.

(B) Let (*) be proved for $1,2, \ldots, n-1$. To prove it for $n$, notice that both sides of $(*)$ are homomorphisms from $\left(P_{n}^{\#}, \varphi\right)$; thus it suffices to show that they coincide on $P_{n}$. Indeed, since for each $\xi$ we have $\alpha_{k+1} \cdot \beta_{k}^{\#}$ $=\beta_{k+1} \cdot \alpha_{k}$ (see 3.1) we get

$$
\begin{aligned}
\eta \cdot \alpha_{n}^{*} \cdot \ldots \cdot \alpha_{1}^{*} \cdot r & =\eta \cdot \alpha_{n}^{*} \cdot\left(\beta_{n-1} \cdot \ldots \cdot \beta_{1}\right)^{\#} \cdot r & & (\text { by induction }) \\
& =\alpha_{n} \cdot \beta_{n-1}^{\#} \cdot\left(\beta_{n-2} \cdot \ldots \cdot \beta_{1}\right)^{\#} \cdot r & & \left(\eta \cdot \alpha_{n}^{*}=\alpha_{n}\right) \\
& =\beta_{n} \cdot \alpha_{n-1} \cdot\left(\beta_{n-2} \cdot \ldots \cdot \beta_{1}\right)^{\# \cdot r} & & \left(a_{n} \cdot \beta_{n-1}^{\#}=\beta_{n} \cdot a_{n-1}\right) \\
& =\beta_{n} \cdot \eta \cdot \alpha_{n-1}^{*} \cdot\left(\beta_{n-2} \cdot \ldots \cdot \beta_{1}\right)^{\#} \cdot r & & \left(\eta \cdot a_{n-1}^{*}=\alpha_{n-1}\right) \\
& =\beta_{n} \cdot \eta \cdot \alpha_{n-1}^{*} \cdot \alpha_{n-2}^{*} \cdot \ldots \cdot \alpha_{1}^{*} \cdot r & & (\text { by induction) } \\
& =\beta_{n} \cdot \eta \cdot\left(\beta_{n-1} \cdot \ldots \cdot \beta_{1}\right)^{\# \cdot r} & & (\text { by induction) } \\
& =\eta \cdot\left(\beta_{n} \cdot \ldots \cdot \beta_{1}\right)^{\# \cdot r} & & \left(\eta \cdot \beta_{n}^{\#}=\beta_{n} \cdot \eta\right) .
\end{aligned}
$$

(b) To prove $\bar{a} \in \mathscr{E}$ we shall exhibit, for every $n=0,1,2, \ldots$, a morphism $\tau_{n}: W_{n} \rightarrow E$ with $\tau_{n} \cdot \bar{\alpha}=w_{n}$. Then, given an $(\mathscr{E}, \mathscr{M})$-factorization $\bar{a}=e \cdot m$, we infer that $\left\{\left(\tau_{n} \cdot e\right) \cdot m\right\}$ is a compatible family for the chain $\left\{k_{n}\right\}$; hence so is $\left\{\tau_{n} \cdot e\right\}$; therefore there exists a $\lambda$ with $\tau_{n} \cdot e=w_{n} \cdot \lambda$ and so

$$
w_{n}=w_{n} \cdot(\lambda \cdot m) \quad \text { for each } n
$$

which implies $\lambda \cdot m=1$. Thus $m$ is an isomorphism and $\bar{\alpha}=e \cdot m^{-1} \in \mathscr{E}$.

(b1) Define a sequence $x_{n}: Q \rightarrow P_{n}$ with

$$
\text { (1) } \quad x_{n+1} \cdot \beta_{n+1}=x_{n} \text { and } x_{n+1} \cdot \alpha_{n+1}=x_{n} \cdot \eta_{P_{n}}
$$

by induction on $n$. First $x_{0}=1_{Q}: Q \rightarrow Q$ and $x_{1}$ is the injection $x_{1}: Q$ $\rightarrow P_{1}=Q+F Q$. The inductive step uses the pullback of $\alpha_{n}, \beta_{n}^{\#}$ :

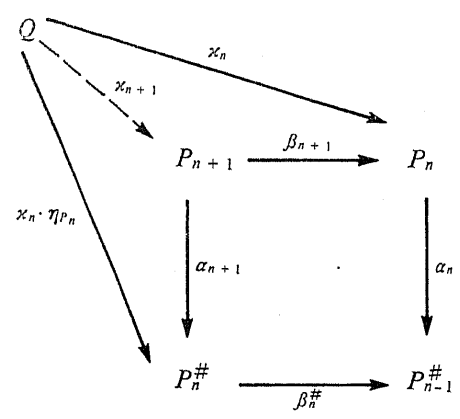


Put $\gamma_{1}=1_{Q}+\delta: Q+F Q \rightarrow Q ; \quad \gamma_{n+1}=1_{Q}+F \gamma_{n}: W_{n+1} \rightarrow W_{n}$. Denote $u_{n}=\eta_{P_{n}}+F \eta_{P_{n}} \cdot \varphi: P_{n}+F P_{n} \rightarrow P_{n}^{\#}$ and define a sequence $\varrho_{n}: W_{n}$ $\rightarrow P_{n}$ with

(2) $\quad \varrho_{n} \cdot \beta_{n}=\gamma_{n} \cdot \varrho_{n-1}$ and $\varrho_{n} \cdot \alpha_{n}=\left(x_{n-1}+F \varrho_{n-1}\right) \cdot u_{n-1}$.

First, $\varrho_{1}=1_{W_{1}}: W_{1} \rightarrow P_{1}=W_{1}$. Given $\varrho_{n}$, let us verify that

$$
\left(\gamma_{n+1} \cdot \varrho_{n}\right) \cdot \alpha_{n}=\left(\varkappa_{n}+F^{\prime} \varrho_{n}\right) \cdot u_{n} \cdot \beta_{n}^{\#}
$$

and then define $\varrho_{n+1}$, using the same pullback as above:

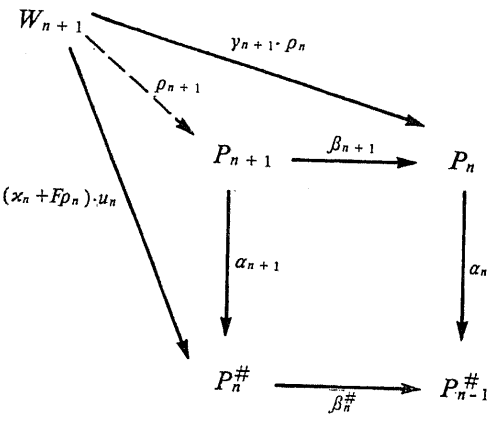

Indeed, since

(4)

we have

$$
u_{n} \cdot \beta_{n}^{\#}=\left(\beta_{n}+F \beta_{n}\right) \cdot u_{n-1}
$$

$$
\begin{array}{rlrl}
\left(\varkappa_{n}+F \varrho_{n}\right) \cdot u_{n} \cdot \beta_{n}^{\#} & =\left(x_{n} \cdot \beta_{n}+F\left(\varrho_{n} \cdot \beta_{n}\right)\right) \cdot u_{n-1} & & (\text { by }(4)) \\
& =\left(x_{n-1}+F\left(\gamma_{n} \cdot \varrho_{n-1}\right)\right) \cdot u_{n-1} & & (\text { by }(1) \text { and }(2)) \\
& =\left(1_{Q}+F \gamma_{n}\right) \cdot\left(x_{n-1}+F \varrho_{n-1}\right) \cdot u_{n-1} & \\
& =\left(1_{Q}+F \gamma_{n}\right) \cdot \varrho_{n} \cdot \alpha_{n} & & \text { (by induction and (2)) } \\
& =\left(\gamma_{n+1} \cdot \varrho_{n}\right) \cdot a_{n} & & \left(\gamma_{n+1}=1_{Q}+F \gamma_{n}\right) .
\end{array}
$$

(b2) Define $\tau_{n}=\varrho_{n} \cdot \bar{e}_{n}: W_{n} \rightarrow E$ (where $\bar{e}_{n}: P_{n} \rightarrow E$ are the colimit injections of $\left.E=\operatorname{colim}\left\{\boldsymbol{e}_{n}\right\}\right)$. Then we prove

$$
\tau_{n} \cdot \bar{a}=w_{n}
$$
by induction. First, $\tau_{1} \cdot \bar{a}=\varrho_{1} \cdot a_{1}=1 \cdot \alpha_{1}=a_{1}=w_{1}$. For the induction
first notice that

$$
u_{n} \cdot \alpha_{n}^{*}=\alpha_{n}+F^{\top} \alpha_{n} \cdot \varphi \quad \text { for each } n
$$

(because in the construction of $P_{n}^{\#}$ as a colimit $w^{\left(P_{n}\right)}: W_{k}^{\left(P_{n}\right)} \rightarrow P_{n}^{\#}$ we have $u_{n}=w_{1}^{\left(P_{n}\right)}$ and $\alpha_{n}^{*}$ is a free extension of $\alpha_{n}$, see 2.1). Further

$$
x_{n} \cdot \eta_{P_{n}} \cdot a_{n}^{*} \cdot \ldots \cdot a_{1}^{*}=\eta_{Q} \quad \text { for each } n
$$

because of (1), which yields $x_{n} \cdot \eta \cdot \alpha_{n}^{*}=x_{n} \cdot \alpha_{n}=x_{n-1} \cdot \eta$. Finally, since $\alpha_{n}^{*} \cdot \ldots \cdot \alpha_{1}^{*}:\left(P^{\#}, \varphi\right) \rightarrow\left(Q^{\#}, \varphi\right)$ is a homomorphism, we have

$$
\varphi \cdot a_{n}^{*} \cdot \ldots \cdot a_{1}^{*}=F\left(a_{n}^{*} \cdot \ldots \cdot a_{1}^{*}\right) \cdot \varphi
$$

Now, assuming $\tau_{n} \cdot \bar{\alpha}=w_{n}$, we get

$$
\begin{aligned}
& \tau_{n+1} \cdot \bar{\alpha}=\varrho_{n+1} \cdot\left(\bar{e}_{n+1} \cdot \bar{\alpha}\right) \quad \text { (definition of } \tau_{n+1} \text { ) } \\
& =\varrho_{n+1} \cdot \alpha_{n+1} \cdot \alpha_{n}^{*} \cdot \ldots \cdot \alpha_{1}^{*} \quad \text { (definition of } \bar{a} \text { ) } \\
& =\left(x_{n}+F \varrho_{n}\right) \cdot u_{n} \cdot a_{n}^{*} \cdot \ldots \cdot a_{1}^{*} \quad \text { (by (2)) } \\
& =\left(x_{n}+F \varrho_{n}\right) \cdot\left(\alpha_{n}+F \alpha_{n} \cdot \varphi\right) \cdot a_{n-1}^{*} \cdot \ldots \cdot a_{1}^{*}(\text { by }(5)) \\
& =\left(x_{n} \cdot \alpha_{n} \cdot \alpha_{n-1}^{*} \cdot \ldots \cdot \alpha_{1}^{*}\right)+\left(F \varrho_{n} \cdot F \alpha_{n} \cdot \varphi \cdot \alpha_{n-1}^{*} \cdot \ldots \cdot \alpha_{1}^{*}\right) \\
& =\eta_{Q}+F^{\prime}\left(\varrho_{n} \cdot \alpha_{n} \cdot \alpha_{n-1}^{*} \cdot \ldots \cdot \alpha_{1}^{*}\right) \cdot \varphi \quad(\text { by }(6) \text { and }(7)) \\
& =\eta_{Q}+F^{\prime}\left(\varrho_{n} \cdot \bar{e}_{n} \cdot \tilde{a}\right) \cdot \varphi \quad \text { (definition of } \bar{a} \text { ) } \\
& =\eta_{Q}+F w_{n} \cdot \varphi \\
& =w_{n+1}
\end{aligned}
$$

This concludes the proof.

\section{The proof of the theorem on analyses}

Extend $i$ and $1_{Q}$ freely to homomorphisms

$$
i^{*}:\left(I^{\#}, \varphi\right) \rightarrow(Q, \delta) \quad \text { and } \quad r=1_{Q}^{*}:\left(Q^{\#}, \varphi\right) \rightarrow(Q, \delta) .
$$

Then for $i^{\#}:\left(I^{\#}, \varphi\right) \rightarrow\left(Q^{\#}, \varphi\right)($ see 1.6$)$ we have

$$
i^{*}=i^{\# \cdot r:\left(I^{\#}, \varphi\right) \rightarrow(Q, \delta)}
$$

because both $i^{*}$ and $r$ are homomorphisms extending $i: I \rightarrow Q$. Consider pullback of $r$ and $t$ (square I) and the pullback of $i^{\#}$ and $v$ (square II):

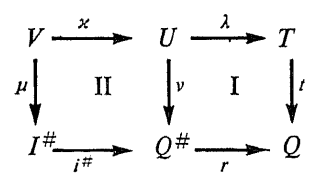

Then the outer square is a pullback of $t$ and $i^{*}=i^{\# \cdot r}$; hence

$$
L(A)=[\mu, x \cdot \lambda]
$$


by Definition 1.5. Further,

$$
[\mu, x \cdot \lambda]=\left[\eta_{I}, i\right] \odot[\nu, \lambda]=L_{A} \odot[\nu, \lambda] .
$$

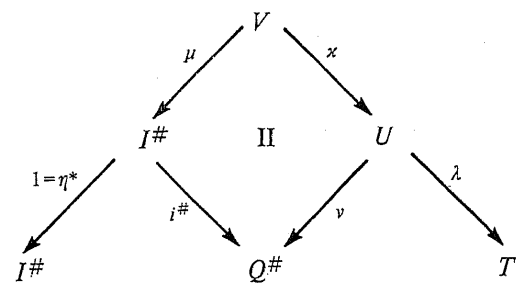

and so, to prove the theorem, it suffices to verify

$$
[v, \lambda]=\left(\Lambda \cup M_{A}\right)^{*} \odot R_{A} .
$$

Now, in the construction of $Q^{\#}(2.1)$ we have $\eta_{Q}=w_{0}$ and $F w_{0} \cdot \varphi=\varphi_{0} \cdot w_{1}$; therefore $w_{1}=\eta_{Q}+F \eta_{Q} \cdot \varphi$. Thus

$\left(\Lambda \cup M_{A}\right)=\left[\eta_{Q}, 1_{Q}\right] \cup\left[F \eta_{Q} \cdot \varphi, \delta\right]=\left[\eta_{Q}+F \eta_{Q} \cdot \varphi, 1_{Q}+\delta\right]=\left[w_{1}, 1_{Q}+\delta\right]$. By 3.3 ,

Hence

$$
\left(\Lambda \cup M_{A}\right)^{*}=\left[1_{Q \#}, r\right]
$$

$$
\begin{aligned}
\left(\Lambda \cup M_{\mathcal{A}}\right)^{*} \odot R_{\mathcal{A}} & =\left[1_{Q \#}, r\right] \odot\left[t \cdot \eta_{Q}, 1_{T}\right]=\left[1_{Q \#}^{*}, r^{\#}\right] \circ\left[t \cdot \eta_{Q}, 1_{T}\right] \\
& =\left[1_{Q \#}^{*}, r^{\#}\right] \circ\left[\eta_{Q}, 1_{Q}\right] \circ\left[t, 1_{T}\right] .
\end{aligned}
$$

The proof will be concluded when we show

$$
\left[1_{Q \#}^{* \#}, r^{\#}\right] \circ\left[\eta_{Q}, 1_{Q}\right]=\left[1_{Q \#}, r\right] .
$$

Consider the pullback of $r^{\#}$ and $\eta_{Q}$ (square $\mathrm{III}$ ):

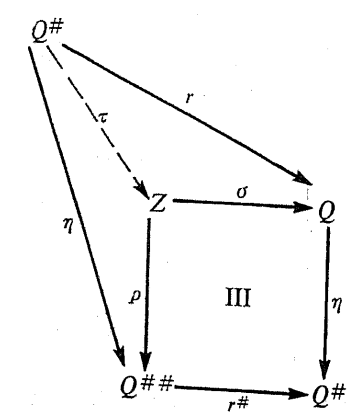

By definition of $r^{\#}$ we have $r \cdot \eta=\eta \cdot r^{\#}$; hence there exists a $\tau: Q^{\#} \rightarrow Z$ with $\tau \cdot \varrho=\eta$. It follows that

$$
\tau \cdot\left(\varrho \cdot 1_{Q \#}^{*}\right)=\eta \cdot 1_{Q \#}^{*}=1_{Q \#} ; \text { thus } 1_{Q \#}^{*} \in \mathscr{E} .
$$

This implies

$$
\left[1_{Q \#}, r\right]=\left[\varrho \cdot 1_{Q \#}^{*}, \varrho \cdot 1_{Q \#}^{*} \cdot r\right] .
$$

Further, both $r \# \cdot r$ and $1_{Q}^{*} \cdot r$ are homomorphisms from $\left(Q^{\# \#}, \varphi\right)$ to $(Q, \delta)$ which equal $r$ on $Q$; hence $r^{\# \cdot r}=1_{Q}^{*} \cdot r$. Therefore $\varrho \cdot 1_{Q \#}^{*} \cdot r=\varrho \cdot r^{\#} \cdot r$ $=\sigma \cdot \eta \cdot r=\sigma($ for $\eta \cdot r=1)$. We get

$$
\left[1_{Q \#}, r\right]=\left[\varrho \cdot 1_{Q \#}^{*}, \sigma\right] \text {. }
$$

This proves $(*)$, because $\left[\varrho \cdot 1_{Q \#}^{*}, \sigma\right]=\left[1_{Q \#}^{*}, r^{\#}\right] \circ\left[\eta_{Q}, 1_{Q}\right]$.

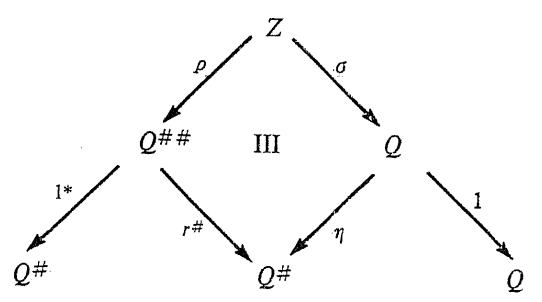

This proves the theorem.

\section{References}

[1] J. Adámek, Free algebras and automata realizations in the language of categories, Comment. Math. Univ. Carolinae 15 (1974), 589-602.

[2] J. Adámek and V. Trnková, Recognizable and regular languages in a category, Lecture Notes Comp. Sci. 56, Springer-Verlag, 1977, 206-211.

[3] -, -, Tarietors and machines, a survey paper, COINS Technical Report, University of Massachusetts, Alg. Universalis 13 (1981), 89-132.

[4] M. A. Arbib and E. G. Manes, Machines in a category, an expository introduction, SIAM Review 16 (1974), 163-192.

[5] M. Barr, P. A. Frillet, and R. van 0 s dob, Exact categories, Lectures Notes Math. 236, Springer-Verlag, 1971.

[6] K. Jarzembski, thesis.

[7] J. W. Thatcher and J. B. Wright, Generalized finite autonomata theory with an application to a decision problem of second order logic, Math. Syst. Theory 2 (1968), 57-81.

[8] V. Trnková and J. Adámek, On languages, accepted by machines in the category of sets, Lecture Notes Comp. Sci. 53, Springer-Verlag, 1977, 523-531. 
[9] V. Trnková, Relational automata in a category and their languages, Lecture WARSAW 1982

Notes Comp. Sci. 56, Springer-Verlag, 1977, 340-355.

[10] -, General theory of relational automata, Fund. Informaticae 3 (1980), 189-233.

Presented to the Semester

Universal Algebra and Applications

(February 15-June 9, 1978)

\section{WEAK AUTOMORPHISMS OF I-UNARY ALGEBRAS}

\author{
LIBOR POLAK
}

Department of Algebra and Geometry, Faculty of Science of JEP University, 66295 Brno, Ozechoslovaliia

Groups of weak automorphisms of 1-unary algebras have been described only in the case of free algebras (see [5] and [1]). Proposition 1 of this note generalizes these results and gives a description for the general case. Proposition 2 tells which groups are of the form "group of all weak automorphisms modulo group of all automorphisms" for 1-unary algebras, Proposition 3 shows that the class of all groups of weak automorphisms of 1-unary algebras is at least as rich as the class of all groups of automorphisms of these, and Proposition 4 ensures that this class does not contain all groups.

I would like to express my thanks for inspiration to my students R. Ptáčník and Z. Svoboda, the authors of [4]. In fact, they have studied groups of weak automorphisms for some 1-unary algebras but without the help of groups of automorphisms of those algebras.

Note that groups of automorphisms of 1-unary algebras were characterized in [2].

For a 1-unary algebra $\mathfrak{A}=(A, f)$ let $\mathscr{A} \mathfrak{U}=(A \mathfrak{A}, \cdot)$ and $\mathscr{W} \mathfrak{A}$ $=(W \mathscr{U}, \cdot)$ denote the group of all automorphisms and the group of all weak automorphisms of it, respectively. Let $f^{n}$ stand for the $n$th iteration of $f$ (i.e. $f^{0}=\operatorname{id}_{A}, f^{n+1} a=f\left(f^{n} a\right)$ for all $\left.a \in A, n=1,2, \ldots\right)$. By $N$ we denote the set of all positive natural numbers and let $\mathscr{Z}_{a}=\left(Z_{d}=\{0,1, \ldots\right.$ $\ldots, d-1\}, \cdot)$ be a semigroup, where the operation $\cdot$ is the usual multiplication modulo $d, d \in N$.

Proposition 1. Let $\mathfrak{A}=(A, f)$ be a 1-unary algebra.

(1) If for no $n \in N f^{n+1}=f$, then any weak automorphism of $\mathfrak{A}$ is an automorphism of $\mathfrak{A}$.

(2) Let $d$ be the smallest $n \in N$ such that $\mathfrak{A}$ satisfies $f^{n+1}=f$. Then the set 